\title{
Article
}

\section{NQO1 is required for beta-lapachone-mediated downregulation of breast cancer stem cell activity}

\author{
Dong Wook Kim ${ }^{1}$ and Je-Yoel Cho ${ }^{1, *}$ \\ Department of Biochemistry, BK21 PLUS Program for Creative Veterinary Science Research and \\ Research Institute for Veterinary Science, College of Veterinary Medicine, Seoul National \\ University, Seoul, South Korea; bellocan@snu.ac.kr (D.W.K); jeycho@snu.ac.kr (J.-Y.C) \\ * Correspondence: jeycho@snu.ac.kr; Tel.: +82-2-880-1268
}

\begin{abstract}
Background: Cancer stem cells (CSCs) exhibit self-renewal activity and give rise to other cell types in tumors. Due to the infinite proliferative potential of CSCs, drugs targeting these cells are necessary to completely inhibit cancer development. beta-lapachone (bL) has been widely used to treat cancer development, but its effect on cancer stem cells remain elusive. Thus, we investigated the effect of bL on mammosphere formation using breast cancer stem cell (BCSC) marker positive cells, MDA-MB-231.

Methods: MDA-MB-231 Cells, which is negative for NQO1 expression, was constructed to stably express NQO1(NQO1 stable cells) to see the effect of bL. The effect of bL on cells were evaluated by wound healing and Transwell cell culture chambers, and ALDEFLUOR assay.

Results: Here, we show that bL inhibited the proliferative ability of mammosphere derived from BCSC marker-positive cells, MDA-MB-231, in an NQO1-dependent manner. bL treatment efficiently downregulated expression level of BCSC markers CD44, ALDH1A1, and DLGAP5 that recently identified as a stem cell proliferation marker in both cultured cells and mammosphered cells. Moreover, bL efficiently downregulates cell proliferation and migration activities.

Conclusions: These results strongly suggest that bL could be a therapeutic agent targeting breast cancer stem cells with proper NQO1 expression.
\end{abstract}

Keywords: beta-lapachone; Breast cancer stem cell; DLGAP5; Mammosphere; NQO1

\section{Introduction}

Cancer stem cells (CSCs) have self-renewal activity, and the population in tumors is very low, ranging from 0.1 to a few percent. Recent studies have identified that CSCs could divide in an asymmetric or symmetric manner depending on the size of the niche [1]. Many studies have advanced the understanding of the biology of breast cancer stem cell (BCSC) development. Stem cells (SCs) in breast tissue also exhibit self-renewal activity and divide into transient amplifying progenitor cells, which become differentiated cells. Of note, BCSCs may originate from stem cells, transient amplifying progenitor cells, and differentiated cells through genetic alteration by environmental stimuli, indicating that the development of BCSCs is a very dynamic process [2]. The population of BCSCs in a tumor is very small and expresses stem cell-associated markers. Well-defined BCSC markers for the identification of BCSCs in tumor tissue and cell lines are CD44 ${ }^{+} / \mathrm{CD}_{24} / \mathrm{ALDH}^{+}$ phenotypes, although other possible human BCSC markers such as $\mathrm{CD} 49 \mathrm{f}^{+}$have been proposed [27]. A characteristic of BCSCs is an enhanced resistance to drugs, radiation, and cell stress that is strongly associated with metastasis and relapse. Four major mechanisms in BCSCs for therapy resistance have been proposed. First, BCSCs have an active ABC transporter system that pumps out 
anthracycline or taxanes, which are two key drugs for breast cancer treatment [8,9]. Second, aldehyde dehydrogenase (ALDH1) activity is highly increased [10]. ALDH1 facilities oxidation of intracellular aldehydes to carboxylic acids and retinoic acids, as well as gamma-amino butyric acid biosynthesis. Moreover, ALDH1 induces radioresistance in BCSCs by direct removal of oxygen radicals and indirect production of the antioxidant compound nicotinamide adenine dinucleotide (NAD). Third, BCSCs have an active DNA double-strand break (DSB) repair system (particularly induced by ionizing radiation), which is repaired by two distinct and complementary systems of homologous recombination (HR) and non-homologous end-joining (NHEJ) [11]. Fourth, BCSCs have specific mechanisms to protect themselves from the genotoxic effects of reactive oxygen species (ROS) by overexpressing ROS scavenging genes such as superoxide dismutase, catalase, and glutathione peroxides $[12,13]$.

Beta-lapachone (bL) is an o-naphthoquinone extracted from plants and has many pharmacological effects [14]. In the last decade, many studies to elucidate the effect of bL on cancer development have been conducted and have shown that bL has an inhibitory effect on various cancers such as epidermoid laryngeal cancer [15] and prostate [16-19], colon [20, 21], ovarian [19], lung[22], and breast cancer [23]. bL has been identified to suppress cancer proliferation by directly interacting with and inhibiting the catalytic activity of DNA topoisomerase I [24], by inducing apoptosis or necrosis by releasing mitochondrial cytochrome C from mitochondria [25] and PARP cleavage [26, 27], by blocking the lethal DNA damage repair (PLDR) system [28], by inducing G1/S cell cycle arrest [29], and by activating c-JUN NH2-terminal kinase [30] and caspases [31]. Recent data have revealed that $\mathrm{NAD}(\mathrm{P}) \mathrm{H}$ :quinone oxidoreductase (NQO1) is a critical enzyme in bLmediated inhibition of cancer proliferation [23, 32]. NQO1 converts cellular NAD $(\mathrm{P}) \mathrm{H}$ into $\mathrm{NAD}(\mathrm{P})^{+}$, and bL accelerates this reaction by accepting hydrogen released from $\mathrm{NAD}(\mathrm{P}) \mathrm{H}$, thereby increasing the cellular level of $\mathrm{NAD}(\mathrm{P})^{+}[33$. Moreover, reduction of bL by NQO1 leads to futile cycling, resulting in the generation of superoxide and hydrogen peroxide [34-36]. In addition, direct reduction of bL by NADPH-cytochrome $\mathrm{p}-450$ reductase (P450R) and NADH-cytochrome b5 reductase (b5R) can generate superoxide and hydrogen peroxide $[37,38]$.

NQO1 is a multifunctional antioxidant enzyme, and its expression and deletion have been closely related with decreased and increased susceptibilities to oxidative stress, respectively [39]. The expression level of NQO1 is highly increased in various cancers, including prostate cancer [38], hepatocellular carcinoma [40], and breast cancer [41, 42], indicating an essential role of NQO1 in cancer development. Oxidative stress is one of the most important regulatory mechanisms for cancer stem cells. Oxidative stress in cancer cells is known to play a key role in the initiation and progression of cancer or in the induction of cancer cell death, depending on the intensity of the oxidative stress $[43,44]$. CSCs and normal stem cells have a low level of ROS, which is an important environment for stem cell maintenance in a stressful environment, meaning that there is high expression of ROS-scavenging molecules [45]. Recent data suggest that nuclear factorerythroid 2-related factor 2 (NRF2), which is a well-known NQO1 upstream regulator, is involved in the maintenance of quiescence, survival, and stress resistance of CSCs [46].

Here, we report that $\beta$-lapachone induces cell death and disruption of mammospheres from breast cancer cells in an NQO1-dependent manner. Our data indicated that bL has an inhibitory effect on the expression of BCSC markers and cell proliferation in an NQO1-dependent manner, suggesting that certain cancer stem cells expressing NQO1 could be a potential therapeutic target of bL.

\section{Results}

\subsection{The cellular expression level of NQO1 is negatively correlated with those of BCSC markers}

At first, we investigated whether NQO1 expression is related to the expression levels of BCSC markers. We compared two breast cancer cell lines, MCF7 and MDA-MB-231, which are known as negative and positive cell lines for BCSC markers, respectively [49] Quantitative RT-PCR indicated that the mRNA expression level of NQO1 is very low in BCSC-marker-positive cells (MDA-MB-231) compared to BCSC-negative cells (MCF-7) (Fig. 1A). Two well-known breast-cancer stem cell 
markers, CD44 and ALDH1A1, were examined. Interestingly, CD44 showed a high expression level in MDA-MB-231 cells (Fig. 1B), but ALDH1A1 did not, although it showed a higher trend (Fig. 1C). In addition, we found that the expression of DLGAP5, which is known to increase stem cell proliferation and be highly expressed in cancers [50-53], was also higher in MDA-MB-231 compared with MCF7 cells (Fig. 1D), indicating that DLGAP5 may positively regulate BSCS proliferation. The protein expression levels of the genes performed for quantitative RT-PCR were verified by western blot analysis. As shown in Fig. 1B, NQO1 was only detected in MCF7 cells. Moreover, CD44 and DLGAP5 were highly expressed in MDA-MB-231 cells, but ALDH1A1 showed no differences or were slightly higher in MDA-MB-231 cells, consistent with the mRNA expression level. Taken together, these results indicated that NQO1 is related to the expression of BCSC markers.

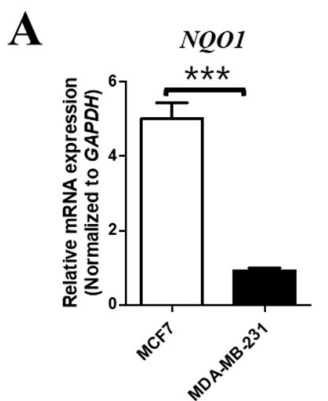

C

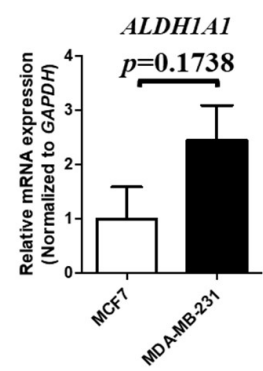

B

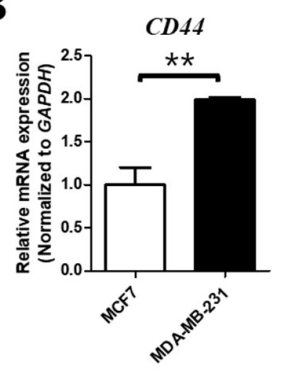

D

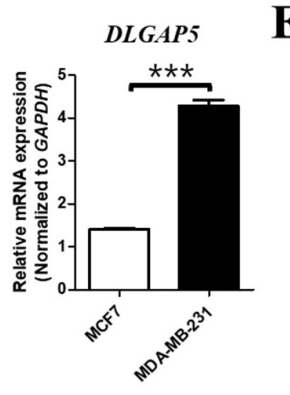

E

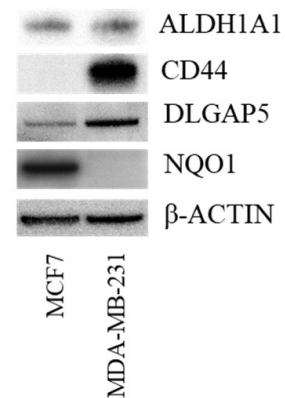

Figure 1. Different expression level of BCSCs markers between MCF7 and MDA-MB-231. (A) The mRNA expression levels of NQO1, DLGAP5, CD44, and ALDH1A1 were compared using quantitative real-time PCR, as described in the Materials and Methods. GAPDH was used as an internal control, and each expression level was normalized to that of GAPDH. The data are presented as the mean \pm SEM. ( $\mathrm{n}=3$; ${ }^{*} \mathrm{p}$-value $<0.05,{ }^{* *}$-value $<0.01,{ }^{* * *} \mathrm{p}$-value $<0.001$, and N.S; nonsignificant). (B) Cell lysates obtained from MCF7 and MDA-MB-231 cells were subjected to western blot analysis to measure the protein expression level of BCSC markers determined by quantitative RT-PCR. $\beta$-ACTIN was used as a loading control.

\subsection{Beta-lapachone-mediated NQO1 activation regulates the DLGAP5, CD44 expression levels.}

To gain insight into the possible mechanism by which NQO1 regulates DLGAP5, CD44 expression, we created MDA-MB-231 cells stably expressing NQO1 (NQO1 stable cells) or the vector control (control cells). The expression of each gene was compared in control cells and in two different clones of NQO1 stable cell lines with or without bL. Interestingly, the gene expression of DLGAP5 and CD44 was downregulated by bL treatment in the presence of NQO1 in MDA-MB-231 cells, but not in control cells, indicating that NQO1 is required for the bL-mediated downregulation of these genes (Fig. 2A and B). In contrast, the ALDH1A1 expression level was not altered by bL treatment regardless of NQO1 expression in both control and NQO1 stable cell lines (Fig. 2C). To verify the effect of NQO1 by bL on protein expression, western blot analysis was performed after bL treatment on control and NQO1 stable cells (Fig. 2D). As expected, bL treatment did not affect the protein expression levels of DLGAP5, CD44, and ALDH1A1 in control cells. Interestingly, the DLGAP5 
protein level was increased by NQO1 exogenous expression alone, and bL treatment dramatically decreased the DLGAP5 protein expression in NQO1 stable cells. Moreover, CD44 expression was not affected by NQO1 expression alone but was still decreased by bL treatment in NQO1 stable cells. These results imply that DLGAP5 and CD44 protein expression could be regulated in various mechanisms by NQO1, and bL is essential for NQO1-mediated regulation of gene and protein expression. Unexpectedly, ALDH1A1 was also downregulated by bL treatment in NQO1 stable cells, which were different from the mRNA expression pattern, suggesting that NQO1 activation by bL might regulate ALDH1A1 expression at the post-translational modification level (Fig. 2D).

A

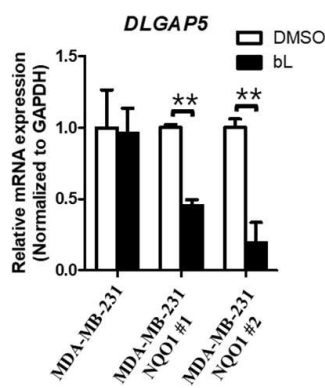

C

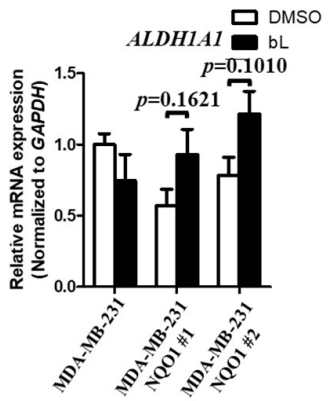

B

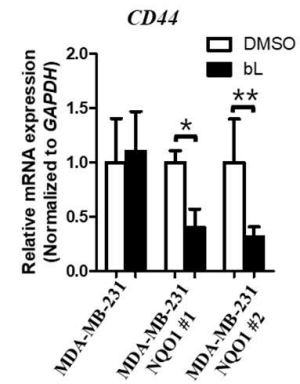

D

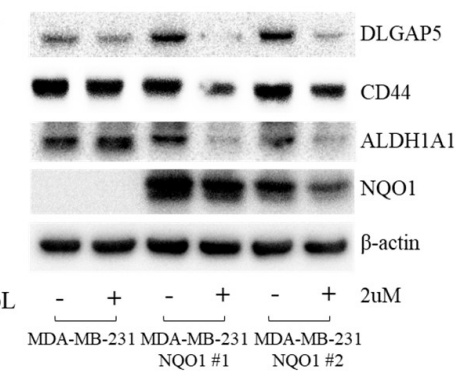

Figure 2. bL suppresses the expression of BCSC markers in an NQO1-dependent manner. (A) The mRNA expression levels of DLGAP5, CD44, and ALDH1A1 were compared among MDA-MB-231 and two independent clones of NQO1 stable cells (NQO1 \#1 and \#2) with or without bL $(2 \mu \mathrm{M})$ for a $24 \mathrm{~h}$ treatment. GAPDH was used as an internal control, and each expression level was normalized to that of GAPDH. The data are presented as the mean \pm SEM. $\left(\mathrm{n}=3\right.$; ${ }^{*} \mathrm{p}$-value $<0.05,{ }^{* *} \mathrm{p}$-value $<0.01,{ }^{* * *} \mathrm{p}$ value $<0.001$, and N.S; Nonsignificant). (B) Protein expression levels of DLGAP, CD44, and ALDH1A1 were compared among MDA-MB-231 and two independent clones of NQO1 stable cells (NQO1 \#1 and \#2) with or without bL $(2 \mu \mathrm{M})$ for a $24 \mathrm{~h}$ treatment. Beta-actin was used as a loading control.

\subsection{SIRT1 is not involved in bL-NQO1-mediated gene expression and cell death.}

SIRT1 is an $\mathrm{NAD}^{+}$dependent deacetylase and regulates gene expression by regulating acetylation on proteins [54]. Because SIRT1 is observed in both the cytosol and nucleus, its localization is regarded as an important event in the regulation of cell proliferation [54]. In addition, NQO1 activated by bL accelerates the conversion of NADH to $\mathrm{NAD}^{+}$, and increased cellular $\mathrm{NAD}^{+}$levels may affect cancer cell proliferation. Therefore, we hypothesized that a cellular $\mathrm{NAD}^{+}$level increased by bL-NQO1 may activate SIRT1 and regulate BCSC marker gene expression. To verify our hypothesis, we first examined SIRT1 cellular localization after bL treatment. Using NQO1 stable cells, we fractionated cells after treatment with bL for $24 \mathrm{hr}$. NQO1 was observed mainly in the cytoplasmic fraction, and DLGAP5 and ALDH1A1 were observed in the nucleus. Notably, the DLGAP5 and ALDH1A1 protein levels were again downregulated by bL treatment in the presence of NQO1 expression (Fig. 3A). However, we could not find any difference in SIRT1 protein amount in the cytoplasmic and nuclear fractions by bL treatment. Moreover, treatment of bL for 15 and $30 \mathrm{~min}$ did not affect SIRT1 localization (Fig. 3B). Finally, we performed tests with a SIRT1 inhibitor to see whether SIRT1 is involved in bL-NQO1-mediated cell survival (Fig. 3C). In control cells, there were 
no differences in cell survival among the bL-only treatment, bL+SIRT1 inhibitor (EX-527) and bL+NQO1 inhibitor (ES 936) groups. As expected, the NQO1 inhibitor, but not the SIRT1 inhibitor, efficiently blocked bL-mediated cell death. These results indicated that SIRT1 is not involved in bLNQO1-mediated cell death.

A

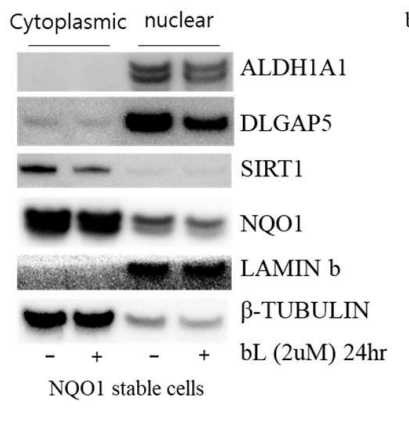

B

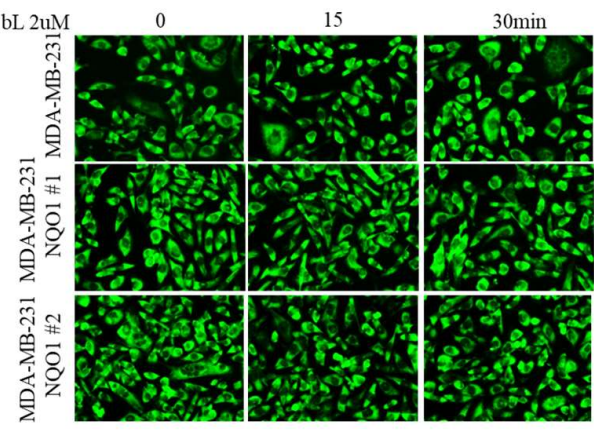

C

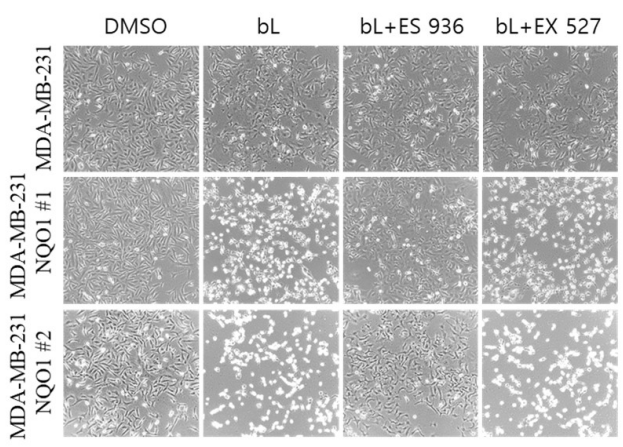

Figure 3. SIRT1 is not involved in bL-NQO1-mediated cell death. (A) Cytoplasmic and nuclear fractionation was performed as described in the Materials and Methods, using NQO1 stable cells with or without bL $(2 \mu \mathrm{M})$ for $24 \mathrm{hr}$. The cell lysate was subjected to western blot analysis with the indicated antibodies. $\beta$-TUBULIN and LAMIN $\mathrm{b}$ were used for cytoplasmic and nuclear markers, respectively. (B) Cellular localization of SIRT1 was examined in MDA-MB-231 and two independent clones of NQO1 stable cells (NQO1 \#1 and \#2) with or without bL $(2 \mu \mathrm{M})$ for the indicated time. Images were taken under a new hybrid microscope (Echo). (C) Cell morphologies of MDA-MB-231 and two independent clones of NQO1 stable cells (NQO1 \#1 and \#2) were examined after treatment with a combination of bL $(2 \mu \mathrm{M})$, an NQO1 inhibitor (ES 936, $1 \mu \mathrm{M})$, and an SIRT1 inhibitor (EX 527, $1 \mu \mathrm{M})$ for $24 \mathrm{~h}$, as indicated. Images were taken using a new hybrid microscope (Echo)

\subsection{NQO1 activated by bL decreases cell proliferation and migration.}

To determine the effect of bL on cell viability, cell proliferation and migration assays were performed with or without bL in control and NQO1 stable cells. For the cell proliferation assay, cell numbers were counted after treatment with bL for $24 \mathrm{hr}$. bL treatment dramatically inhibited cell proliferation in NQO1 stable cells (Fig. 4A). Notably, NQO1 expression alone did not affect cell proliferation, as there was no difference among cell lines without bL treatment. Wound healing (Fig. 4B) and migration assays using a transwell system (Fig. 4C) showed that bL treatment significantly decreased the cell migration ability at indicated times. Importantly, NQO1 expression alone did not show any effect on cell migration, which is consistent with the results of the cell proliferation assay. Taken together, these results indicated that bL increased the NQO1-mediated inhibition of cell proliferation and migration. 
A

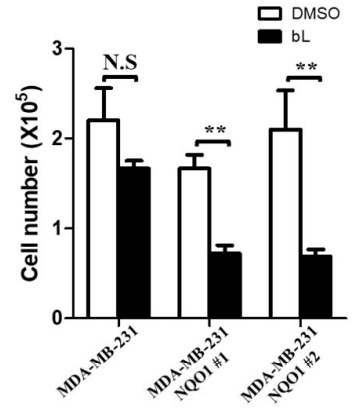

B

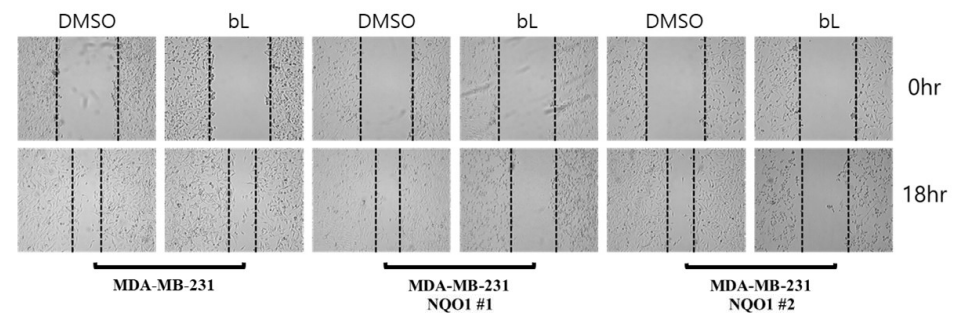

C

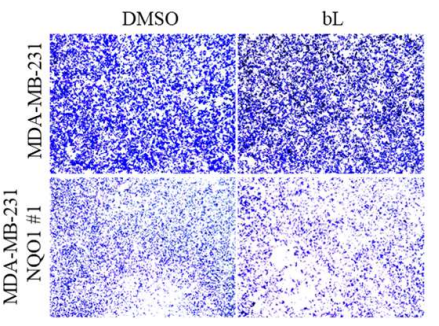

Figure 4. bL suppresses cell proliferation and migration in an NQO1-dependent manner. (A) Cell proliferation was determined by counting the cell numbers after treatment with bL $(2 \mu \mathrm{M})$ for $24 \mathrm{~h}$ using MDA-MB-231 and two independent clones of NQO1 stable cells (NQO1 \#1 and \#2). The data are presented as the mean \pm SEM. $\left(n=3 ;{ }^{*}\right.$-value $<0.05,{ }^{* *}$-value $<0.01,{ }^{* * *}$-value $<0.001$, and N.S; nonsignificant). (B) A wound healing assay was performed as described in the Materials and Methods, using MDA-MB-231 and two independent clones of NQO1 stable cells (NQO1 \#1 and \#2) treated with bL $(2 \mu \mathrm{M})$ for $18 \mathrm{hr}$. Images were taken using a new hybrid microscope (Echo). (C) A cell migration assay was performed with Transwell cell culture chambers as described in the Materials and Methods, using MDA-MB-231 and NQO1 stable cells treated with bL ( $2 \mu \mathrm{M})$ for $7 \mathrm{hr}$. Images were taken using a new hybrid microscope (Echo).

\subsection{ALDH1 activity is decreased by bL through NQO1.}

The bL treatment decreased the ALDH1A1 protein expression, but not mRNA, level in an NQO1-dependent manner (Fig. 2C \& 2D). Therefore, we investigated whether NQO1 activated by bL treatment could directly affect the activity of ALDH1. To this end, we took advantage of the ALDEFLUOR assay system. Using a cell permeable substrate of ALDH1, endogenous ALDH1 activity was evaluated according to the intensity of green fluorescence (Fig. 5A). Cells with weak green fluorescence (Aldefluor-low) was slightly increased in control cells by bL treatment. However, bL significantly increased Aldefluor-low cells in NQO1 stable cells. Quantification analysis identified that bL treatment increased Aldefluor-low cells approximately 2-fold in control cells and approximately 3.7-fold and 3.4-fold in NOQ1 stable cells, respectively (Fig. 5B). These results indicated that bL in the presence of NQO1 could decrease endogenous ALDH1 activity. 
A

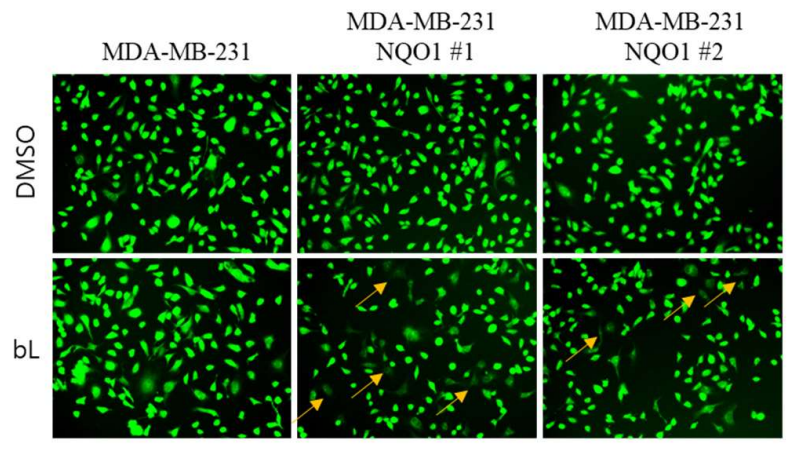

B

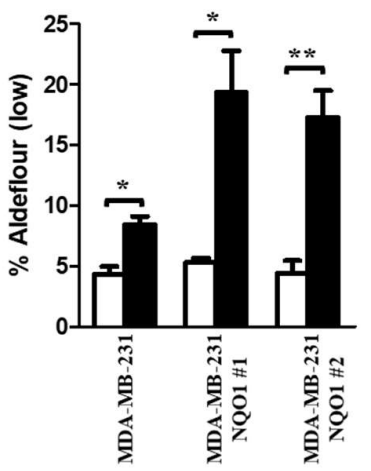

Figure 5. bL decreases endogenous ALDH1 activity. The overall activity of ALDH1 was examined using an ALDEFLUOR assay in MDA-MB-231 and two independent clones of NQO1 stable cells (NQO1 \#1 and \#2) treated with bL $(2 \mu \mathrm{M})$ for $24 \mathrm{hr}$. Green fluorescence was captured under a new hybrid microscope (Echo). For quantification, three independent areas were randomly taken, and cells with a lower intensity of ALDEFLUOR than the DMSO control were counted. Quantitative analysis revealed that bL efficiently decreased the endogenous activity of ALDH1 in an NQO1dependent manner. The yellow arrow indicates ALDEFLUOR (low) cells.

\subsection{Mammosphere formation was inhibited by bL-NQO1.}

bL through NQO1 was shown to efficiently inhibit the proliferation of MDA-MB-231 cells, which are positive for BCSC markers, and decreased the expression and activity of CD44 and ALDH1A1, respectively. In addition, DLGAP5 expression that was known to increase stem cell proliferation was downregulated by bL treatment. These observations lead us to test whether bL could suppress mammosphere formation. Cells were cultured in mammosphere media for 1 or 2 weeks, and a round type of stem-cell-like phenotype was observed in 2-week culture (Fig. 6A). Interestingly, NQO1 expression alone seemed to increase the size of mammospheres compared with control cells. Strikingly, the size of mammospheres formed after culturing for 1 or 2 weeks was apparently decreased by bL treatment. Next, we tested whether the BCSC markers that we measured in previous results were also altered. Marker protein expression levels were analyzed in the cell lysates obtained from the cells used in Fig. 6A. CD44, DLGAP5, and ALDH1A1 expression levels were dramatically decreased by bL treatment in NQO1-expressing cells (Fig. 6B). Notably, NQO1 alone did not show any effect on these protein expression levels, again as shown in previous results. Taken together, these results suggested that bL-mediated inhibition of mammosphere formation required NQO1 expression. 
A

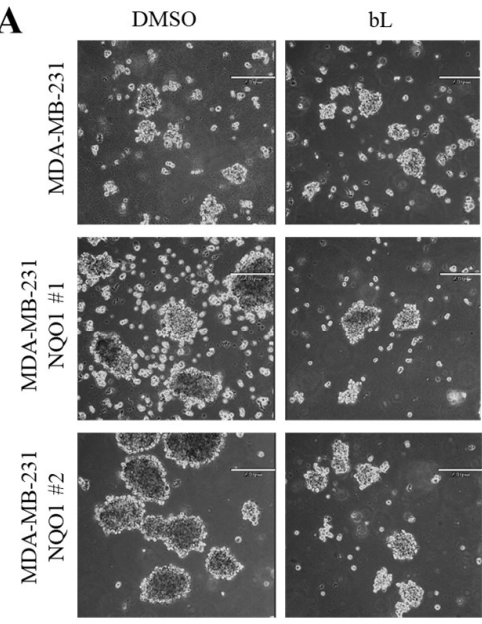

2 weeks
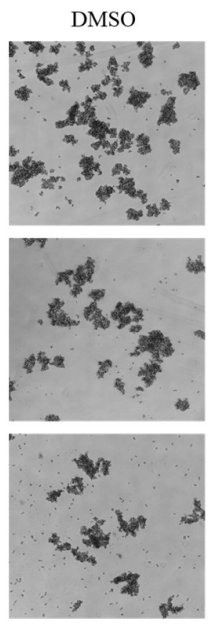

B
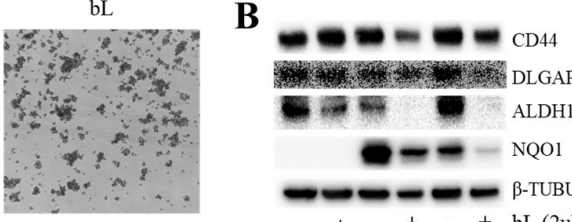

W.1.
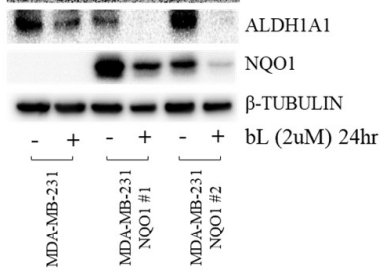

Figure 6. bL inhibits mammosphere formation. (A) MDA-MB-231 and two independent clones of NQO1 stable cells (NQO1 \#1 and \#2) were induced to mammosphere formation for 1 and 2 weeks, as described in the Materials and Methods. After mammosphere formation, bL $(2 \mu \mathrm{M})$ was added for 24 hr. Images were taken using a new hybrid microscope (Echo). (B) Cell lysates obtained from the mammosphere formation assay were subjected to western blot analysis using the indicated antibodies. Beta-tubulin was used as a loading control.

\section{Discussion}

In this paper, we reported that bL could decrease BSCS marker expression and inhibit the mammosphere-formation ability of breast cancer cells in an NQO1-dependent manner. Interestingly, NQO1 expression alone did not affect BSCS marker expression, cell proliferation, and migration. In addition, we also identified that the expression of DLGAP5, which is known to stimulate stem cell proliferation, was decreased by bL treatment in an NQO1-dependent manner. The expression levels of CD44 and DLGAP5, but not ALDH1A1, are regulated at the transcriptional level. The activity of ALDH1A1 was reduced by bL treatment, as evidenced by the ALDEFLUOR assay, meaning that bL has multiple inhibition mechanisms for cell proliferation and survival.

In an attempt to find a downstream mediator of NQO1, we considered a major metabolite produced by NQO1, specifically, $\mathrm{NAD}^{+}$. $\mathrm{NAD}^{+}$is a major cellular metabolite that regulates gene expression and protein activation by activating the sirtuin family [55]. The sirtuin family has been identified as a major target of NAD ${ }^{+}$. Among the sirtuin family members, SIRT1 has been widely studied in cancer development [56]. Unlike other sirtuin family members, SIRT1 can shuttle between the nucleus and cytoplasm and has been reported to be a tumor suppressor or tumor promoter in various cancers [56]. Therefore, we tested to see whether SIRT1 is a key mediator in bL-NQO1induced cell death. Unexpectedly, the cell fractionation experiment and fluorescence microscope analysis showed that SIRT1 localization was not altered by bL treatment even though the protein expression level of DLGAP5 and ALDH1A1 was still decreased by bL treatment. These results mean that there is an alternative cell death pathway induced by bL-NQO1 in breast cancer, although many reports suggest that the NAD-SIRT1 axis could play a key suppressor in cancer proliferation and survival. One of the possible mechanisms of bL-NQO1-mediated cell death is by hydrogen peroxide. Indeed, bL could generate superoxide and hydrogen peroxide by virtue of NADPH-cytochrome p450 reductase and NADH-cytochrome b5 reductase as well as NQO1 [27, 35-37, 39]. Increased hydrogen peroxide may activate PARP and caspase 3 , which has already been identified in other studies [27, 31].

CD44 and ALDH1A11 have been used as positive BCSC markers, and their expression level was downregulated by bL treatment in an NQO1-dependent manner. Interestingly, a decreased expression level of CD44 expression was observed at both the mRNA and protein levels; therefore, 
CD44 expression is regulated at the transcriptional level by bL. In contrast, ALDH1A1 expression is only downregulated at the protein expression level, meaning that bL may regulate post-translational modification. In addition, we found that the expression level of DLGAP5, which has been known to increase stem cell proliferation, was dramatically decreased by bL treatment. DLGAP5 has been identified to be upregulated in hematopoietic progenitor cells and was proposed to play an important role in the proliferation of stem cells [50]. Meanwhile, other studies also identified that DLGAP5 was detected in various cancers, including hepatocellular carcinoma and ovarian cancer cells [52, 57], suggesting its potential role as a fetal oncoprotein. Therefore, bL-mediated downregulation of CD44, ALDH1A1, and DLGAP5 could be an essential mechanism to inhibit cancer cell proliferation, even though the suppression mechanism by bL is different.

To determine the effect of bL on BCSC proliferation in vitro, we took advantage of the mammosphere formation assay. Using BCSC marker-positive cells, specifically, MDA-MB-231 cells, we first induced mammosphere formation. Mammosphere formation is a suitable method to evaluate stem-cell-like features of breast cancer cells. Compared with a 1-week culture, a 2-week culture was sufficient to induce the formation of large mammospheres; subsequently, bL was added to the media. bL-induced inhibition of mammosphere formation was clearly evident in both 1- and 2-week-culture cells. Since SIRT1 was not involved in bL-NQO1 mediated cell death in our experiments, another possible pathway to induce cell death and to inhibit mammosphere formation may be a robust increase in hydrogen peroxide. In our mammosphere formation experiment, we found that NQO1 stable cells without bL treatment induced the formation of slightly larger mammospheres than did control cells. Indeed, several lines of evidence have identified that increased NQO1 expression in cancer stem cells may increase stem cell proliferation and survival by removing excess hydrogen peroxide. Therefore, the key cellular mechanism in bL-induced cell death is to robustly increase the endogenous level of hydrogen peroxide to an extent that cells cannot remove.

\section{Materials and Methods}

\subsection{Cell culture and Stable cell line establishment}

Two human breast cancer cell lines, MCF7 (KCLB No. 30022) and MDA-MB-231 (KCLB No. 30026) were purchased from Korean cell line bank (Seoul, South Korea) and cultured in DMEM with $10 \% \mathrm{FBS}$ in a humidified incubator at $37^{\circ} \mathrm{C}$ and $5 \% \mathrm{CO}_{2}$. Cells used in this study has been tested for mycoplasma contamination. An NQO1-negative cell line, MDA-MB-231, was transiently transfected with an NQO1 expression vector (pEFIRES-NQO1) or a control vector containing a puromycin (Sigma-Aldrich, MO) selection marker using Lipofectamine 3000 reagent (Thermo Scientific, PA) according to the manufacturer's instructions. After $24 \mathrm{~h}$ transfection, the cell medium was changed to a medium containing puromycin at a final concentration of $2 \mu \mathrm{g} / \mathrm{ml}$ every two days. Two independent cell clones with puromycin resistance were used for the experiments.

\subsection{Quantitative Real-Time PCR}

Total RNA was isolated from MCF7 and MDA-MB-231 cells using an RNeasy kit (QIAGEN, Germany) as per the manufacturer's instructions. Quantitative real-time RT-PCR was performed as previously reported [47]. The primers used were as follows: NQO1 forward, 5'AAAGGACCCTTCCGGAGTAA-3'; NQO1 reverse, 5'-CCATCCTTCCAGGATTTGAA-3'; DLGAP5 forward, 5'-CCAGTCGACACAGGAAGGAT-3'; DLGAP5 reverse, 5'CATTGCCCTTGGCTTAACAT-3'; CD44 forward, 5'-TGGACAGGACAGGACCTCTT-3'; CD44 reverse, 5' $5^{\prime} \quad$ AGGTCCTGCTTTCCTTCGTG-3'; ALDH1A1 forward, 5' TTCGAAGGAGTGTTGAGCGG -3'; ALDH1A1 reverse, 5'-AACACTGTGGGCTGGACAAA-3'; and GAPDH forward, 5'-TCCAAAATCAAGTGGGGCGA-3'; GAPDH reverse, 5'ATGACGAACATGGGGGCATC-3'. GAPDH was used as the reference for normalization of each sample. 
10 of 14

\subsection{Western blot analysis}

Western blot analysis was performed as previously reported [48]. Briefly, whole cell lysates were prepared in RIPA buffer, and isolated total proteins were subjected to SDS-PAGE and membrane transfer for the incubation of the following primary antibodies: Anti-NQO1 Ab (Abcam, United Kingdom), CD44 (Abcam, United Kingdom), ALDH1A1 (Abcam, United Kingdom), DLGAP5 (Abcam, United Kingdom), $\beta$-ACTIN (Sigma-Aldrich, MO), SIRT1 (Santa Cruz, TX), Lamin b (Santa Cruz, TX), and $\beta$-TUBULIN (AbFrontier, South Korea). The membrane was developed using a chemiluminescence detection system (ATTO corporation, Japan) and exposed to an X-ray film.

\subsection{Immunocytochemistry}

NQO1 stable cells were seeded into a 6-well plate and treated with bL (Santa Cruz, TX) at a final concentration of $2 \mu \mathrm{M}$ with a vehicle control (DMSO) for $24 \mathrm{~h}$, otherwise indicated. After treatment with bL, the cells were fixed with $4 \%$ paraformaldehyde for $10 \mathrm{~min}$ at room temperature (RT), washed with PBS three times, and then permeabilized with $0.1 \%$ Triton X-100 for $10 \mathrm{~min}$ at RT. The cells were incubated with primary SIRT1 antibodies (1:100) for $3 \mathrm{~h}$ at RT, followed by incubation with fluorescein-labeled goat anti-mouse IgG antibody (Thermo Scientific, PA). Cell images were captured under a new hybrid microscope (Echo, CA).

\subsection{Wound healing assay}

Control and NQO1 stable cells were seeded into a 6-well plate containing the culture medium (DMEM supplemented with 10\% FBS). After $24 \mathrm{~h}$, the cells were wounded with a p200 tip, washed with $1 \times$ PBS, and then treated with bL $(2 \mu \mathrm{M})$ and DMSO. Pictures were taken at the $18 \mathrm{~h}$ time point after bL treatment.

\subsection{ALDEFLUOR assay}

An ALDEFLUOR assay was performed using an ALDEFLUOR kit (Stemcell Technologies, MA) according to the manufacturer's instruction. Briefly, control and NQO1 stable cells were seeded into a 6-well plate and cultured for $24 \mathrm{~h}$ at $37{ }^{\circ} \mathrm{C}$ in an incubator supplied with humidified air containing $5 \% \mathrm{CO}_{2}$. Twenty-four hours later, bL $(2 \mu \mathrm{M})$ was added to the culture media, and the cells were cultured an additional $24 \mathrm{~h}$; next, $10 \mu \mathrm{l}$ of activated ALDEFLUOR was added to the media for $45 \mathrm{~min}$. The cells were washed with PBS three times, and pictures were taken under a hybrid microscope (Echo, CA).

\subsection{Migration assay}

Cell migration activity was tested using a 5.0- $\mu$ m-pore polycarbonate membrane insert Transwell cell culture chambers (Corning, USA). Cells $\left(6 \times 10^{4}\right)$ of control and NQO1 stable lines were seeded into the upper chamber with or without bL $(2 \mu \mathrm{M})$. After incubation for $7 \mathrm{~h}$ at $37{ }^{\circ} \mathrm{C}$, cells on the lower surface were fixed with $4 \%$ paraformaldehyde for $5 \mathrm{~min}$. The cells were washed with PBS three times and stained with $1 \%$ crystal violet in $2 \%$ ethanol for 20 min; next, the cells were washed with PBS three times. Pictures were taken under a microscope.

\subsection{Mammosphere formation}

Mammosphere formation was performed using MammoCult media (Stemcell Technologies) supplemented with $4 \mu \mathrm{g} / \mathrm{ml}$ of heparin and $0.48 \mu \mathrm{g} / \mathrm{ml}$ of hydrocortisone, according to the manufacturer's instructions. In brief, $4 \times 10^{4}$ cells of control and NQO1 stable cells were seeded into ultralow adherent 6-well plates (Stemcell Technologies)) and incubated with MammoCult media for 1 or 2 weeks. Then, bL $(2 \mu \mathrm{M})$ was added for $24 \mathrm{~h}$, and the cell morphology was observed under a hybrid microscope (Echo, CA). 


\subsection{Cytoplasmic and nuclear fractionation}

NQO1 stable cells were seeded into a 100-mm culture dish, cultured for $24 \mathrm{~h}$, and then treated with bL $(2 \mu \mathrm{M})$ for an additional $24 \mathrm{hr}$. Cytoplasmic and nuclear fractionation was performed using a NE-PER Nuclear and Cytoplasmic Extraction Kit (Thermo Scientific, PA) according to the manufacturer's instruction. Beta-tubulin and Lamin b were used as cytoplasmic and nuclear markers, respectively.

\subsection{Statistical analyses}

Data are expressed as the mean \pm standard error of the mean. Significant differences between groups were calculated using Student's $t$-test via GraphPad Prism 5 (GraphPad Software, CA). $P<0.05$ was considered statistically significant.

\section{Conclusions}

In this studies, we found that bL selectively inhibits the proliferation of mammosphere by downregulating CD44, DLGAP5, and ALDH1A1 in a NQO1 dependent manner. These results strongly suggest that $b \mathrm{~L}$ could be a potential therapeutic agent targeting breast cancer stem cells with proper NQO1 expression.

Author Contributions: D.K.W designed and performed cell experiments, statistical analyses, made the figures, and wrote the manuscript. J.-Y.C helped in interpretation of data and wrote the manuscript.

Funding: This work was supported by the National Research Foundation (NRF) funded by the Ministry of Science, ICT \& Future Planning (2016M3A9B6026771, 2014M3A9D5A01073598) and by the Basic Science Research Program through the National Research Foundation of Korea (NRF) funded by the Ministry of Education (2017R1D1A1B03030844).

Conflicts of Interest: The authors declare no conflict of interest. The funders had no role in the design of the study; in the collection, analyses, or interpretation of data; in the writing of the manuscript, or in the decision to publish the results.

\section{Abbreviations}

bL

NQO1 NAD $(\mathrm{P}) \mathrm{H}$ quinone dehydrogenase 1

CSCs cancer stem cells

BCSCs breast cancer stem cells

CD44 CD44 molecules

DLGAP5 DLG-associated protein 5

ALDH1A1 aldehyde dehydrogenase 1 family member A1

SIRT1 sirtuin 1

NAD nicotinamide adenine dinucleotide

ROS reactive oxygen species

\section{References}

1. Batlle, E.; Clevers, H., Cancer stem cells revisited. Nature medicine 2017, 23, (10), 1124-1134.

2. Sin, W. C.; Lim, C. L., Breast cancer stem cells-from origins to targeted therapy. Stem cell investigation 2017, 4,96 .

3. Lagadec, C.; Vlashi, E.; Della Donna, L.; Dekmezian, C.; Pajonk, F., Radiation-induced reprogramming of breast cancer cells. Stem Cells 2012, 30, (5), 833-44.

4. Koren, S.; Reavie, L.; Couto, J. P.; De Silva, D.; Stadler, M. B.; Roloff, T.; Britschgi, A.; Eichlisberger, T.; Kohler, H.; Aina, O.; Cardiff, R. D.; Bentires-Alj, M., PIK3CA(H1047R) induces multipotency and multilineage mammary tumours. Nature 2015, 525, (7567), 114-8. 
5. Kim, H. J.; Kim, M. J.; Ahn, S. H.; Son, B. H.; Kim, S. B.; Ahn, J. H.; Noh, W. C.; Gong, G., Different prognostic significance of CD24 and CD44 expression in breast cancer according to hormone receptor status. Breast 2011, 20, (1), 78-85.

6. Moreb, J. S.; Ucar, D.; Han, S.; Amory, J. K.; Goldstein, A. S.; Ostmark, B.; Chang, L. J., The enzymatic activity of human aldehyde dehydrogenases 1A2 and 2 (ALDH1A2 and ALDH2) is detected by Aldefluor, inhibited by diethylaminobenzaldehyde and has significant effects on cell proliferation and drug resistance. Chemico-biological interactions 2012, 195, (1), 52-60.

7. Lo, P. K.; Kanojia, D.; Liu, X.; Singh, U. P.; Berger, F. G.; Wang, Q.; Chen, H., CD49f and CD61 identify Her2/neu-induced mammary tumor-initiating cells that are potentially derived from luminal progenitors and maintained by the integrin-TGFbeta signaling. Oncogene 2012, 31, (21), 2614-26.

8. Nakanishi, T.; Ross, D. D., Breast cancer resistance protein (BCRP/ABCG2): its role in multidrug resistance and regulation of its gene expression. Chinese journal of cancer 2012,31, (2), 73-99.

9. Carrasco, E.; Alvarez, P. J.; Prados, J.; Melguizo, C.; Rama, A. R.; Aranega, A.; Rodriguez-Serrano, F., Cancer stem cells and their implication in breast cancer. European journal of clinical investigation 2014, 44, (7), 67887.

10. Charafe-Jauffret, E.; Ginestier, C.; Bertucci, F.; Cabaud, O.; Wicinski, J.; Finetti, P.; Josselin, E.; Adelaide, J.; Nguyen, T. T.; Monville, F.; Jacquemier, J.; Thomassin-Piana, J.; Pinna, G.; Jalaguier, A.; Lambaudie, E.; Houvenaeghel, G.; Xerri, L.; Harel-Bellan, A.; Chaffanet, M.; Viens, P.; Birnbaum, D., ALDH1-positive cancer stem cells predict engraftment of primary breast tumors and are governed by a common stem cell program. Cancer research 2013, 73, (24), 7290-300.

11. van Oorschot, B.; Granata, G.; Di Franco, S.; Ten Cate, R.; Rodermond, H. M.; Todaro, M.; Medema, J. P.; Franken, N. A., Targeting DNA double strand break repair with hyperthermia and DNA-PKcs inhibition to enhance the effect of radiation treatment. Oncotarget 2016, 7, (40), 65504-65513.

12. Czerwinska, P.; Kaminska, B., Regulation of breast cancer stem cell features. Contemp Oncol (Pozn) 2015, 19, (1A), A7-A15.

13. Diehn, M.; Cho, R. W.; Lobo, N. A.; Kalisky, T.; Dorie, M. J.; Kulp, A. N.; Qian, D.; Lam, J. S.; Ailles, L. E.; Wong, M.; Joshua, B.; Kaplan, M. J.; Wapnir, I.; Dirbas, F. M.; Somlo, G.; Garberoglio, C.; Paz, B.; Shen, J.; Lau, S. K.; Quake, S. R.; Brown, J. M.; Weissman, I. L.; Clarke, M. F., Association of reactive oxygen species levels and radioresistance in cancer stem cells. Nature 2009, 458, (7239), 780-3.

14. Pardee, A. B.; Li, Y. Z.; Li, C. J., Cancer therapy with beta-lapachone. Current cancer drug targets 2002, 2, (3), 227-42.

15. Boothman, D. A.; Greer, S.; Pardee, A. B., Potentiation of halogenated pyrimidine radiosensitizers in human carcinoma cells by beta-lapachone (3,4-dihydro-2,2-dimethyl-2H-naphtho[1,2-b]pyran- 5,6-dione), a novel DNA repair inhibitor. Cancer research 1987, 47, (20), 5361-6.

16. Costa, M. P.; Feitosa, A. C.; Oliveira, F. C.; Cavalcanti, B. C.; da Silva, E. N.; Dias, G. G.; Sales, F. A.; Sousa, B. L.; Barroso-Neto, I. L.; Pessoa, C.; Caetano, E. W.; Di Fiore, S.; Fischer, R.; Ladeira, L. O.; Freire, V. N., Controlled Release of Nor-beta-lapachone by PLGA Microparticles: A Strategy for Improving Cytotoxicity against Prostate Cancer Cells. Molecules 2016, 21, (7).

17. Dong, Y.; Bey, E. A.; Li, L. S.; Kabbani, W.; Yan, J.; Xie, X. J.; Hsieh, J. T.; Gao, J.; Boothman, D. A., Prostate cancer radiosensitization through poly(ADP-Ribose) polymerase-1 hyperactivation. Cancer research 2010, 70, (20), 8088-96.

18. Li, C. J.; Wang, C.; Pardee, A. B., Induction of apoptosis by beta-lapachone in human prostate cancer cells. Cancer research 1995, 55, (17), 3712-5.

19. Li, C. J.; Li, Y. Z.; Pinto, A. V.; Pardee, A. B., Potent inhibition of tumor survival in vivo by beta-lapachone plus taxol: combining drugs imposes different artificial checkpoints. Proceedings of the National Academy of Sciences of the United States of America 1999, 96, (23), 13369-74.

20. Kee, J. Y.; Han, Y. H.; Park, J.; Kim, D. S.; Mun, J. G.; Ahn, K. S.; Kim, H. J.; Um, J. Y.; Hong, S. H., betaLapachone Inhibits Lung Metastasis of Colorectal Cancer by Inducing Apoptosis of CT26 Cells. Integrative cancer therapies 2017, 16, (4), 585-596.

21. Liu, H.; Li, Q.; Cheng, X.; Wang, H.; Wang, G.; Hao, H., UDP-glucuronosyltransferase $1 \mathrm{~A}$ determinates intracellular accumulation and anti-cancer effect of beta-lapachone in human colon cancer cells. PloS one 2015, 10, (2), e0117051.

22. Bey, E. A.; Bentle, M. S.; Reinicke, K. E.; Dong, Y.; Yang, C. R.; Girard, L.; Minna, J. D.; Bornmann, W. G.; Gao, J.; Boothman, D. A., An NQO1- and PARP-1-mediated cell death pathway induced in non-small-cell 
lung cancer cells by beta-lapachone. Proceedings of the National Academy of Sciences of the United States of America 2007, 104, (28), 11832-7.

23. Yang, Y.; Zhou, X.; Xu, M.; Piao, J.; Zhang, Y.; Lin, Z.; Chen, L., beta-lapachone suppresses tumour progression by inhibiting epithelial-to-mesenchymal transition in NQO1-positive breast cancers. Scientific reports 2017, 7, (1), 2681.

24. Li, C. J.; Averboukh, L.; Pardee, A. B., beta-Lapachone, a novel DNA topoisomerase I inhibitor with a mode of action different from camptothecin. The Journal of biological chemistry 1993, 268, (30), 22463-8.

25. Li, Y. Z.; Li, C. J.; Pinto, A. V.; Pardee, A. B., Release of mitochondrial cytochrome C in both apoptosis and necrosis induced by beta-lapachone in human carcinoma cells. Mol Med 1999, 5, (4), 232-9.

26. Breton, C. S.; Aubry, D.; Ginet, V.; Puyal, J.; Heulot, M.; Widmann, C.; Duchosal, M. A.; Nahimana, A., Combinative effects of beta-Lapachone and APO866 on pancreatic cancer cell death through reactive oxygen species production and PARP-1 activation. Biochimie 2015, 116, 141-53.

27. Park, E. J.; Min, K. J.; Lee, T. J.; Yoo, Y. H.; Kim, Y. S.; Kwon, T. K., beta-Lapachone induces programmed necrosis through the RIP1-PARP-AIF-dependent pathway in human hepatocellular carcinoma SK-Hep1 cells. Cell death $\mathcal{E}$ disease 2014, 5, e1230.

28. Boothman, D. A.; Pardee, A. B., Inhibition of radiation-induced neoplastic transformation by betalapachone. Proceedings of the National Academy of Sciences of the United States of America 1989, 86, (13), 49637.

29. Jeon, Y. J.; Bang, W.; Shin, J. C.; Park, S. M.; Cho, J. J.; Choi, Y. H.; Seo, K. S.; Choi, N. J.; Shim, J. H.; Chae, J. I., Downregulation of Sp1 is involved in beta-lapachone-induced cell cycle arrest and apoptosis in oral squamous cell carcinoma. International journal of oncology 2015, 46, (6), 2606-12.

30. Shiah, S. G.; Chuang, S. E.; Chau, Y. P.; Shen, S. C.; Kuo, M. L., Activation of c-Jun NH2-terminal kinase and subsequent CPP32/Yama during topoisomerase inhibitor beta-lapachone-induced apoptosis through an oxidation-dependent pathway. Cancer research 1999, 59, (2), 391-8.

31. Yu, H. Y.; Kim, S. O.; Jin, C. Y.; Kim, G. Y.; Kim, W. J.; Yoo, Y. H.; Choi, Y. H., beta-lapachone-Induced Apoptosis of Human Gastric Carcinoma AGS Cells Is Caspase-Dependent and Regulated by the PI3K/Akt Pathway. Biomolecules \& therapeutics 2014, 22, (3), 184-92.

32. Silvers, M. A.; Deja, S.; Singh, N.; Egnatchik, R. A.; Sudderth, J.; Luo, X.; Beg, M. S.; Burgess, S. C.; DeBerardinis, R. J.; Boothman, D. A.; Merritt, M. E., The NQO1 bioactivatable drug, beta-lapachone, alters the redox state of NQO1+ pancreatic cancer cells, causing perturbation in central carbon metabolism. The Journal of biological chemistry 2017, 292, (44), 18203-18216.

33. Dong, Y.; Chin, S. F.; Blanco, E.; Bey, E. A.; Kabbani, W.; Xie, X. J.; Bornmann, W. G.; Boothman, D. A.; Gao, J., Intratumoral delivery of beta-lapachone via polymer implants for prostate cancer therapy. Clinical cancer research: an official journal of the American Association for Cancer Research 2009, 15, (1), 131-9.

34. Pink, J. J.; Planchon, S. M.; Tagliarino, C.; Varnes, M. E.; Siegel, D.; Boothman, D. A., NAD(P)H:Quinone oxidoreductase activity is the principal determinant of beta-lapachone cytotoxicity. The Journal of biological chemistry 2000, 275, (8), 5416-24.

35. Chau, Y. P.; Shiah, S. G.; Don, M. J.; Kuo, M. L., Involvement of hydrogen peroxide in topoisomerase inhibitor beta-lapachone-induced apoptosis and differentiation in human leukemia cells. Free radical biology $\mathcal{E}$ medicine 1998, 24, (4), 660-70.

36. Cruz, F. S.; Docampo, R.; Boveris, A., Generation of superoxide anions and hydrogen peroxide from betalapachone in bacteria. Antimicrobial agents and chemotherapy 1978, 14, (4), 630-3.

37. Rauth, A. M.; Goldberg, Z.; Misra, V., DT-diaphorase: possible roles in cancer chemotherapy and carcinogenesis. Oncology research 1997, 9, (6-7), 339-49.

38. Planchon, S. M.; Pink, J. J.; Tagliarino, C.; Bornmann, W. G.; Varnes, M. E.; Boothman, D. A., betaLapachone-induced apoptosis in human prostate cancer cells: involvement of NQO1/xip3. Experimental cell research 2001, 267, (1), 95-106.

39. Dinkova-Kostova, A. T.; Talalay, P., NAD(P)H:quinone acceptor oxidoreductase 1 (NQO1), a multifunctional antioxidant enzyme and exceptionally versatile cytoprotector. Archives of biochemistry and biophysics 2010, 501, (1), 116-23.

40. Lin, L.; Sun, J.; Tan, Y.; Li, Z.; Kong, F.; Shen, Y.; Liu, C.; Chen, L., Prognostic implication of NQO1 overexpression in hepatocellular carcinoma. Human pathology 2017, 69, 31-37. 
41. Glorieux, C.; Sandoval, J. M.; Dejeans, N.; Ameye, G.; Poirel, H. A.; Verrax, J.; Calderon, P. B., Overexpression of $\mathrm{NAD}(\mathrm{P}) \mathrm{H}$ :quinone oxidoreductase 1 (NQO1) and genomic gain of the NQO1 locus modulates breast cancer cell sensitivity to quinones. Life sciences 2016, 145, 57-65.

42. Yang, Y.; Zhang, Y.; Wu, Q.; Cui, X.; Lin, Z.; Liu, S.; Chen, L., Clinical implications of high NQO1 expression in breast cancers. Journal of experimental \& clinical cancer research: CR 2014, 33, 14.

43. Dayem, A. A.; Choi, H. Y.; Kim, J. H.; Cho, S. G., Role of oxidative stress in stem, cancer, and cancer stem cells. Cancers 2010, 2, (2), 859-84.

44. Reuter, S.; Gupta, S. C.; Chaturvedi, M. M.; Aggarwal, B. B., Oxidative stress, inflammation, and cancer: how are they linked? Free radical biology \& medicine 2010, 49, (11), 1603-16.

45. Shi, X.; Zhang, Y.; Zheng, J.; Pan, J., Reactive oxygen species in cancer stem cells. Antioxidants $\mathcal{E}$ redox signaling 2012, 16, (11), 1215-28.

46. Ryoo, I. G.; Lee, S. H.; Kwak, M. K., Redox Modulating NRF2: A Potential Mediator of Cancer Stem Cell Resistance. Oxidative medicine and cellular longevity 2016, 2016, 2428153.

47. Lee, B. N. R.; Son, Y. S.; Lee, D.; Choi, Y. J.; Kwon, S. M.; Chang, H. K.; Kim, P. H.; Cho, J. Y., HedgehogInteracting Protein (HIP) Regulates Apoptosis Evasion and Angiogenic Function of Late Endothelial Progenitor Cells. Scientific reports 2017, 7, (1), 12449.

48. Choi, Y. J.; Jeong, S.; Yoon, K. A.; Sung, H. J.; Cho, H. S.; Kim, D. W.; Cho, J. Y., Deficiency of DGCR8 increases bone formation through downregulation of miR-22 expression. Bone 2017, 103, 287-294.

49. Li, W.; Ma, H.; Zhang, J.; Zhu, L.; Wang, C.; Yang, Y., Unraveling the roles of CD44/CD24 and ALDH1 as cancer stem cell markers in tumorigenesis and metastasis. Scientific reports 2017, 7, (1), 13856.

50. Gudmundsson, K. O.; Thorsteinsson, L.; Sigurjonsson, O. E.; Keller, J. R.; Olafsson, K.; Egeland, T.; Gudmundsson, S.; Rafnar, T., Gene expression analysis of hematopoietic progenitor cells identifies Dlg7 as a potential stem cell gene. Stem Cells 2007, 25, (6), 1498-506.

51. Loo, L. W.; Cheng, I.; Tiirikainen, M.; Lum-Jones, A.; Seifried, A.; Dunklee, L. M.; Church, J. M.; Gryfe, R.; Weisenberger, D. J.; Haile, R. W.; Gallinger, S.; Duggan, D. J.; Thibodeau, S. N.; Casey, G.; Le Marchand, L., cis-Expression QTL analysis of established colorectal cancer risk variants in colon tumors and adjacent normal tissue. PloS one 2012, 7, (2), e30477.

52. Tsou, A. P.; Yang, C. W.; Huang, C. Y.; Yu, R. C.; Lee, Y. C.; Chang, C. W.; Chen, B. R.; Chung, Y. F.; Fann, M. J.; Chi, C. W.; Chiu, J. H.; Chou, C. K., Identification of a novel cell cycle regulated gene, HURP, overexpressed in human hepatocellular carcinoma. Oncogene 2003, 22, (2), 298-307.

53. Eissa, S.; Matboli, M.; Mansour, A.; Mohamed, S.; Awad, N.; Kotb, Y. M., Evaluation of urinary HURP mRNA as a marker for detection of bladder cancer: relation to bilharziasis. Med Oncol 2014, 31, (2), 804.

54. Choupani, J.; Mansoori Derakhshan, S.; Bayat, S.; Alivand, M. R.; Shekari Khaniani, M., Narrower insight to SIRT1 role in cancer: A potential therapeutic target to control epithelial-mesenchymal transition in cancer cells. Journal of cellular physiology 2018, 233, (6), 4443-4457.

55. Zhang, N.; Sauve, A. A., Regulatory Effects of NAD(+) Metabolic Pathways on Sirtuin Activity. Progress in molecular biology and translational science 2018, 154, 71-104.

56. Lin, Z.; Fang, D., The Roles of SIRT1 in Cancer. Genes \& cancer 2013, 4, (3-4), 97-104.

57. Chen, X.; Thiaville, M. M.; Chen, L.; Stoeck, A.; Xuan, J.; Gao, M.; Shih Ie, M.; Wang, T. L., Defining NOTCH3 target genes in ovarian cancer. Cancer research 2012, 72, (9), 2294-303. 